\section{Pancreatoduodenectomy for trauma: applying novel reconstruction techniques}

\author{
Jake E.J. Krige, ${ }^{1-3}$ Sandie R. Thomson ${ }^{4,5}$ \\ 'Surgical Gastroenterology Unit; \\ ${ }^{2}$ Hepatobiliary and Pancreatic Surgical \\ Unit, Groote Schuur Hospital, Cape \\ Town; ${ }^{3}$ Department of Surgery, \\ University of Cape Town Health Sciences \\ Faculty, Cape Town; ${ }^{4}$ Medical \\ Gastroenterology Unit, Groote Schuur \\ Hospital, Cape Town; ${ }^{5}$ Department of \\ Medicine, University of Cape Town \\ Health Sciences Faculty, Cape Town, \\ South Africa
}

hypothermia, coagulopathy and acidosis which compound the physiological derangement. ${ }^{1,2}$ In addition, technical difficulties resecting and reconstructing complex pancreatic injuries require special surgical skills and expertise..$^{1,3}$ Although other reports ${ }^{2,4,5}$ and from our own institution $^{6-8}$ have detailed aspects of the management of pancreatic injuries including the overall management of proximal ${ }^{3,9}$ and distal resection, ${ }^{10}$ no publications have specifically assessed the technical aspects of reconstruction after emergency PD for complex injuries of the pancreas and duodenum. This study evaluated the outcome of pancreatic, biliary and gastric reconstruction methods after PD for major pancreatic injuries in a cohort of consecutive patients treated at a level 1 academic trauma center.

\section{Materials and Methods}

This single center study evaluated the technical modifications and outcome of reconstruction after pancreaticoduodenectomy for trauma. Prospectively recorded data including reconstructive techniques used in patients who underwent a pancreatoduodenectomy (PD) for trauma were analyzed. Twenty patients underwent a PD. Six had an initial damage control procedure. Thirteen had a pylorus-preserving PD and 7 a standard Whipple resection because injury to the pylorus precluded a pylorus-preserving resection. Twelve patients had a pancreatojejunostomy and 8 a pancreatogastrostomy, 3 of whom had a duodenojejunal hepaticojejunal sequence of anastomoses to allow endoscopic biliary stent retrieval. Three patients died postoperatively of multi-organ failure. All 17 survivors had postoperative complications: 5 patients developed pancreatic fistula, 2 had gastric outlet obstruction, 2 had bile leaks, 2 had duodenal anastomotic leaks, all of which resolved with conservative treatment. Pancreatic and biliary reconstructions performed under adverse conditions after a trauma PD required a variety of technical modifications. The pylorus does not have to be sacrificed and posterior gastric implantation is a safe option for an edematous pancreas.

\section{Introduction}

Pancreatoduodenectomy (PD) and subsequent reconstruction after complicated pancreatic injuries in severely injured patients has in the past resulted in prohibitive mortality rates. Exsanguination due to collateral injuries involving the inferior vena cava, portal or superior mesenteric veins frequently result in

\section{Study population and data collection}

The study design was a single center retrospective cohort analysis of prospective data on consecutive patients who had a PD for complex combined pancreatic and duodenal injuries between January 1990 and December 2014. The study used a registered fit for purpose departmental database, which documents the details of all patients with pancreatic injuries treated at the level 1 Trauma Centre and the Hepatopancreatobiliary and Surgical Gastroenterology units at Groote Schuur Hospital, Cape Town. Data relating to each patient were entered prospectively on a standardised electronic password protected Microsoft Access data spread sheet and analyzed using Microsoft Access and Microsoft Excel. All data collected were validated by a trained surgical clinical reviewer. For the purposes of this study all clinical records including operative, intensive care, radiology, endoscopic and multidisciplinary clinic reports of patients with pancreatic injuries were accessed from the database and reviewed. Variables recorded included patient demographic data, mechanism, site, indices of severity of the injury and methods of reconstruction. Pancreas-related and other complications, duration of hospital stay and mortality were also documented. Other aspects of pancreatic injury management using this database have been published. ${ }^{6-8,10}$ The study was approved by the University of Cape Town, Human Research Ethics Committee.

\section{Definitions}

All patients who had a PD had grade 5 pancreatic injuries according to the Organ Injury Scaling (OIS) of the American Association for
Correspondence: Jake E.J. Krige, Department of Surgery, University of Cape Town Health Sciences Faculty, Anzio Road, Observatory 7925 , Cape Town, South Africa.

Tel: +27.214043072 - Fax: +27.214480981 .

E-mail: jej.krige@.uct.ac.za

Key words: Pancreatoduodenectomy; Trauma; Reconstruction.

Conflict of interest: the authors declare no potential conflict of interest.

Contributions: JEJK is the main author and responsible for the study design, data analysis, data interpretation, literature search, writing and critical revision. SRT is responsible for data analysis, data interpretation, writing and critical revision.

Received for publication: 3 November 2015 .

Revision received: 15 April 2016.

Accepted for publication: 22 April 2016.

This work is licensed under a Creative Commons Attribution NonCommercial 4.0 License (CC BYNC 4.0).

(C) Copyright J.E.J. Krige and S.R. Thomson, 2016 Licensee PAGEPress, Italy

Surgical Techniques Development 2016; 6:6293 doi:10.4081/std.2016.6293

the Surgery of Trauma (AAST). ${ }^{11}$ Morbidity was considered for systemic, intra-abdominal and specific complications directly related to the pancreatic injury. Pancreatic fistulas were evaluated according to the International Study Group of Pancreatic Fistula ${ }^{12}$ and postoperative complications were classified according to the Clavien-Dindo grading system. ${ }^{13}$ Grade $3-6$ complications were considered as severe. Mortality was defined as any cause of death in hospital after a pancreatic injury.

\section{Operative technique of pancreatic reconstruction}

Details outlining our management and operative strategies of pancreatic injuries have been published. ${ }^{1,6-8,10} \mathrm{~A}$ pylorus preserving pancreatoduodenectomy (PPPD) was undertaken in all patients in whom the injury had not irretrievably damaged the pylorus. In those requiring a gastric resection a classic Whipple resection was done. The pancreatic remnant was mobilized for $2.5 \mathrm{~cm}$ from the splenic and portal vein confluence to facilitate the pancreatic anastomosis (Figure 1). The end-to-side pancreatojejunostomy was constructed by placing the inferior row of interrupted $3 / 0$ polydioxanone (PDS) sutures to include the edge of the pancreas and incorporate capsule and parenchyma as well as full thickness jejunal wall. In those in whom the pancreatic duct could be identified, the anastomosis was stent- 
ed internally with an $8 \mathrm{~cm} 5 \mathrm{Fr}$ silastic pediatric feeding tube cut to size. Into the pancreatic duct were inserted $4 \mathrm{~cm}$ of stent and the remaining $4 \mathrm{~cm}$ were placed in the jejunum (Figure 2). The anterior layer pancreatic sutures were individually inserted first and then sequentially into the jejunal wall to complete the anastomosis. In patients in whom the jejunum was grossly edematous after prolonged resuscitation and unsuitable for an anastomosis, the pancreatic stump was drained into the stomach. A 3-cm oblique gastrostomy was made in the posterior wall of the stomach prior to placing interrupted 3/0 PDS sutures first through the edge of the superior surface of the pancreas, incorporating capsule and parenchyma, and then through the full thickness of the superior edge of the gastrostomy. The anastomosis was completed by suturing the posterior margin of the pancreas to the inferior margin of the gastrostomy in a similar manner.

\section{Operative technique of biliary reconstruction}

The biliary anastomosis was a modification of the standard method used for bile duct reconstruction after iatrogenic injuries. ${ }^{14,15}$ The duct was spatulated to increase anastomotic size using an anterior vertical incision

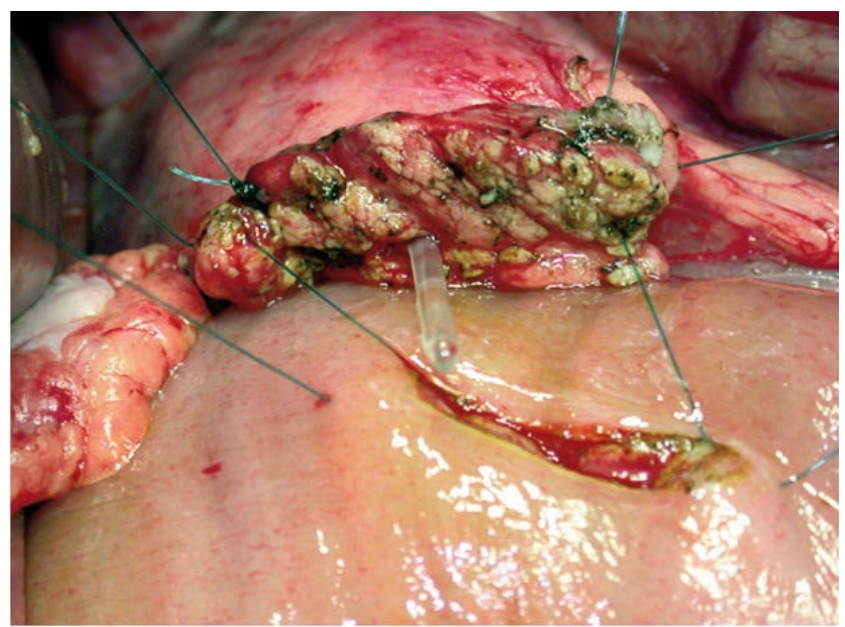

Figure 1. Photograph of a stented end-to-side pancreaticojejunostomy.

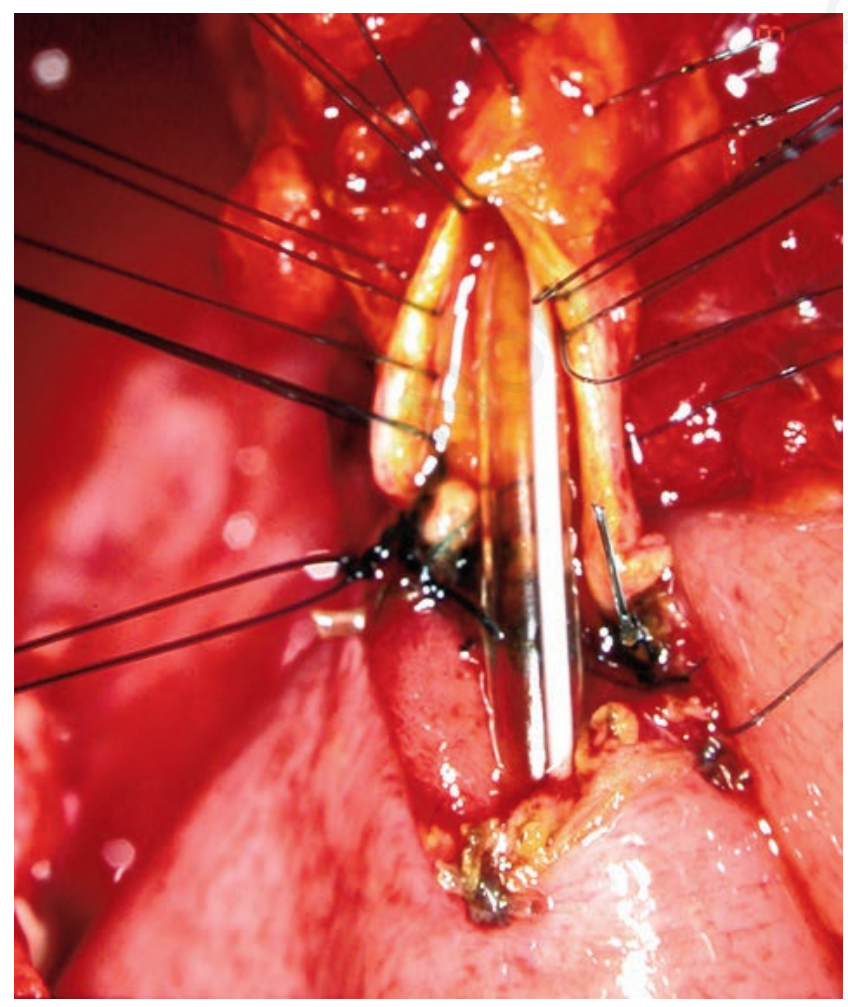

Figure 3. Photograph of a spatulated and stented end-to-side hepaticojejunostomy.

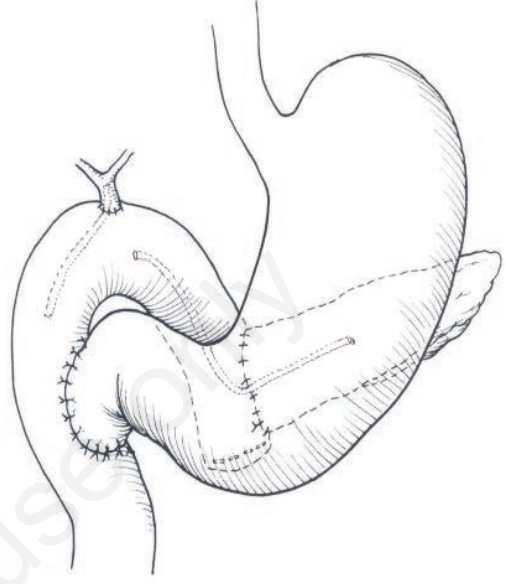

Figure 2. Pylorus preserving pancreatoduodenectomy with a distal duodenojejunostomy and stented pancreatic and biliary jejunal anastomoses.

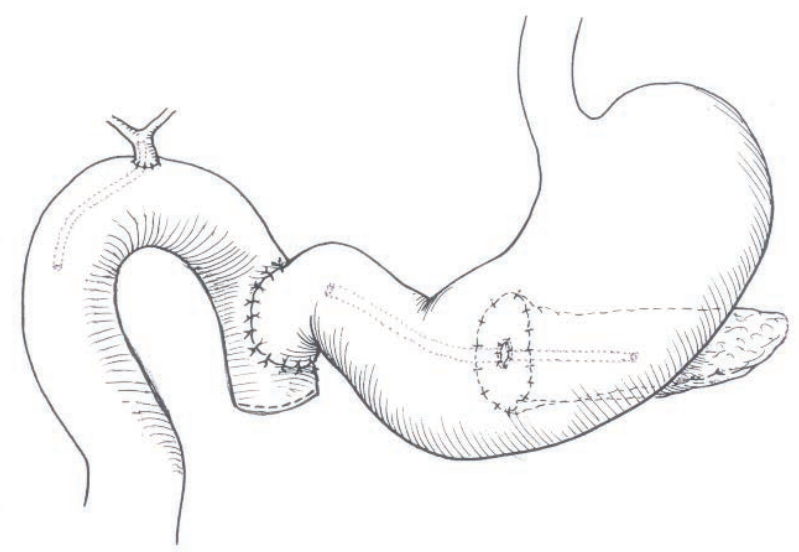

Figure 4. Pylorus preserving pancreatoduodenectomy and modified Imanaga reconstruction with proximal duodenojejunostomy and pancreatogastrostomy. 
positioned to avoid the 3 and 9 o'clock bile duct arteries (Figure 3). All biliary anastomoses were stented with an $8 \mathrm{~cm}$ long $5 \mathrm{Fr}$ silastic pediatric feeding tube. In situations where the bile duct measured less than $3 \mathrm{~mm}$ in width and gross edema jeopardized the bile duct to jejunum anastomosis, the gall bladder was preserved and used as the conduit for the biliaryenteric anastomosis. In high-risk stented biliary anastomoses a modified Imanaga reconstruction technique (Figure 4) was used in which the duodenojejunostomy was created end-to-side as the most proximal jejunal anastomosis to allow post-operative endoscopic retrograde cholangiopancreatography (ERCP) and biliary stent retrieval. ${ }^{16}$

\section{Results}

Twenty (18 men, 2 women, median age 28 years, range 14-53 years) of 426 patients had AAST grade 5 injuries involving the head of the pancreas and duodenum which were not reconstructable and required a PD. Fourteen of the 20 had penetrating injuries (13 gunshot wounds, 1 stab wound) and 6 had sustained blunt abdominal injuries due to motor vehicle accidents. Ten of the 20 patients had 1 or more associated vascular injuries involving inferior vena cava (IVC) $(n=9)$, portal vein $(n=2)$, superior mesenteric vein $(n=2)$, renal veins $(n=3)$ and lumbar veins $(n=1)$. Concurrent extra-abdominal trauma occurred in three patients.

\section{Surgery}

\section{Initial damage control surgery}

In 6 patients, the injury complex produced extreme physiological derangement, which mandated a damage control operation initially, and a subsequent PD and reconstruction. Five of the 6 were shocked on admission to hospital and five had associated vascular injuries. The PD was done at a median of 19 hours (range 11-48 hours) after the initial damage control laparotomy in four patients or during a third laparotomy 48 and 96 hours later in two patients. ${ }^{1}$

\section{Pancreatoduodenal resection}

Thirteen patients had a PPPD and seven a standard Whipples resection (Figure 5). In three patients the reconstruction used a modified Imanaga sequence of anastomosis (Figure 4) to allow for a postoperative ERCP to be performed to access the biliary system for retrieval or replacement of a biliary stent placed because of an associated liver and intrahepatic ductal injury. In three patients the gallbladder was used as the conduit for biliary drainage into the jejunum. Eight patients had a pancreatogastrostomy and in 12 patients an end-to-side pancreatojejunostomy was used (Figure 5). The median duration of intensive care was 5 days (range 1-20 days). Seventeen patients survived. Median duration of hospital stay for survivors was 29 days (range 14-94 days).

\section{Post-operative course}

\section{Morbidity}

One patient had a Clavien-Dindo grade 1 complication, 7 (13.8\%) patients had grade 2 complications, 2 (17.5\%) had grade $3 \mathrm{a}, 6$ (10\%) had grade $4 \mathrm{a}$, and 3 (16.3\%) had grade 5 complications and died. Two patients had an anastomotic leak, two had a bile leak and three

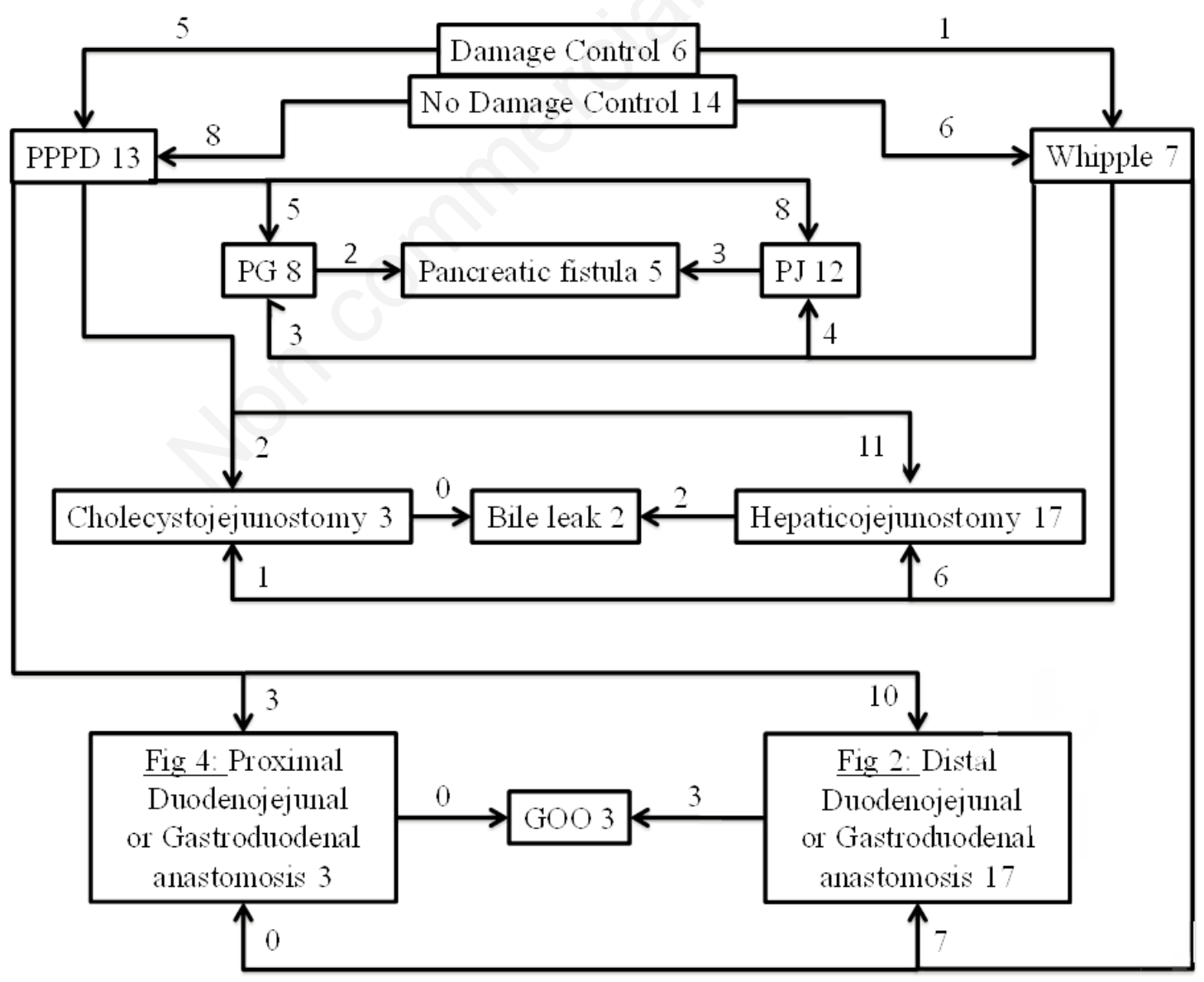

Figure 5. Pancreatoduodenectomy for trauma: operative management, reconstruction and complications. 
had delayed gastric emptying (Figure 5). Five patients developed a pancreatic fistula after the PD, three of twelve patients who had a pancreatojejunostomy and two of eight patients who had a pancreatogastrostomy (Figure 5). All five were grade B fistulas and were treated with fine-bore nasojejunal feeding and octreotide and all resolved after a median of 22 (IQR 12-38) days.

\section{Late complications}

One patient required hospitalization on three occasions with self-limiting alcoholinduced acute pancreatitis and one patient had symptomatic malabsorption, which resolved on pancreatic replacement therapy. Three patients subsequently underwent further surgery. One patient had closure of a de-functioning colostomy 6 months after the PD and two patients in whom the gallbladder had been preserved and used for biliary drainage returned 3 and 6 years after the PD with cholangitis due to hepatic duct stones. Both had a cholecystectomy and a hepaticojejunostomy.

\section{Mortality}

Three of the 20 patients died. All three were profoundly shocked with major splanchnic venous injuries and had APACHE II scores on admission of 15, 18 and 18. Two died of multi organ failure (MOF) and disseminated intravascular coagulopathy within 48 hours after receiving a median of 27 units of blood during the damage control operation. The third patient died after 24 days of MOF due to resistant acinetobacter and pseudomonas intraabdominal sepsis.

\section{Discussion}

This study reports the technical details and outcome of pancreatic, biliary and gastric reconstruction after PD for trauma. There is consensus that a PD for trauma and the subsequent reconstruction is the most taxing of all pancreatic resections because the procedure is undertaken under adverse conditions with severe operative constraints. While some authors recommend that a PD for trauma should be always performed as a two-stage procedure $^{2,17,18}$ our data suggest otherwise. In this study all patients who were hemodynamically stable after intra-operative resuscitation underwent PD and immediate reconstruction with survival of 13 of 14 patients. In contrast, a cohort of six other patients who remained hypotensive despite sustained resuscitation, had an initial damage control operation for hemostasis and staple closure of injured hollow viscera and external pancreatic and biliary drainage. The resection and anastomoses in this latter group were completed when the patient was stable at either the second or third operation.

When the trauma pancreatoduodenal resection has been accomplished, several critical decisions are necessary regarding the timing and method of reconstruction. ${ }^{4}$ The key determinant of the ultimate outcome is the integrity of the pancreatic anastomosis. As in elective resections, the pancreatic anastomosis following a trauma PD is the weakest link and pancreatic anastomotic failure is the most important factor responsible for the substantial postoperative morbidity and mortality. Even under elective circumstances the fistula rate is appreciable and is highest in those with a soft pancreas and a small duct. ${ }^{19}$ These risk factors pertain in the trauma situation and are compounded by a pancreas that is damaged and edematous, as well as an edematous bowel wall, making the situation even more unfavorable for a successful anastomosis..$^{9,20,21}$

In the elective setting a number of different methods have been proposed to reduce the incidence of post-operative pancreatic fistulas including the site of implantation (stomach or jejunum), anastomotic technique and pancreatic duct stenting. ${ }^{22-24}$ These techniques need to be adapted to the prevailing operative circumstances. In this study the solution to overcoming these considerable technical difficulties was to use a stented single layer interrupted anastomosis with a pancreatic leak rate of $30 \%$ in survivors.

A pancreaticogastrostomy (PG) was used in this study when profound shock, prolonged resuscitation and major vascular injuries resulted in an edematous jejunum, which jeopardized the anastomosis. Under these adverse circumstances there are several cogent practical and technical reasons for doing a PG in preference to a pancreatojejunostomy. ${ }^{25,26}$ The posterior wall of the stomach is conveniently positioned adjacent and anterior to the pancreatic remnant and the gastrostomy can be fashioned to the exact size required without any discrepancy in dimensions to allow a tensionfree anastomosis. In addition, the stomach wall is thick, holds sutures well, has an abundant blood supply and is less likely than a jejunal loop to develop ischemic complications. ${ }^{27}$ Gastric and pancreatic secretions are easily removed via a nasogastric tube after PG and the pancreatic exocrine enzymes remain inactivated with a low $\mathrm{pH}$ and in the absence of enterokinase. ${ }^{28}$ As with the pancreaticojejunostomy (PJ), we used a single layer interrupted suture technique incorporating pancreatic capsule and parenchyma with a 5 Fr intraluminal duct stent rather than the more complex and time consuming duct to mucosa technique. PG and PJ each have their own advantages and limitations in elective resections but neither are universally applicable after major pancreatic trauma where edema and tissue damage are crucial factors determining the use a particular anastomosis in the reconstruction. Current evidence suggests that it is technically more important to identify the subgroup of patients who may benefit from a specific technique (PG or PJ) than pursuit a universal technique and attempt a one size fits all methodology. A recent meta-analysis of randomized controlled trials in patients who had either a PG or PJ after an elective PD reported that the pancreatic fistula rate was significantly less in patients who had a PG while there were no differences in delayed gastric emptying, enteric or biliary fistulae, remnant pancreatitis or wound complications when comparing the two reconstruction methods. ${ }^{29}$

An end-to-side hepaticojejunostomy, using the high bile duct reconstruction technique with preplaced sutures ${ }^{14,15}$ is regarded as the gold standard for elective restoration of biliaryenteric continuity. The major risk factors for bile leaks and ultimately biliary strictures are the size of the bile duct and the adequacy of the blood supply of the bile duct. ${ }^{30,31}$ Skinny ducts have been shown to result in bile leaks in $4 \%$ of elective PD reconstructions. ${ }^{30}$ Small size was a universal feature in this study, which required a modified approach and biliary stenting. In patients who had labile vital signs and a small bile duct the gall bladder was used for the anastomosis after ligating the bile duct below the cystic duct insertion. However, two of the three required a revision 3 and 6 years later.

Unlike other reported series, a unique aspect of this study was the capability to do a PPPD in those injured patients in whom the pylorus was intact. Two immediate advantages of a PPPD were retention of the stomach thus allowing the full posterior gastric wall to be accessible for a PG and the modified Imanaga sequence of reconstruction allowed postoperative endoscopic access through the duodenojejunal anastomosis to the biliary system for retrieval of biliary stents and balloon enhanced cholangiography, which was important in those patients who had an associated bile leak due to a gunshot injury of the liver.

\section{Conclusions}

This analysis describes for the first time how techniques for pancreatic, biliary and gastric anastomoses need to be modified for reconstruction after a PD for trauma. Although a relevant limitation of this study is the small sample size, a robust feature of the data accrual and analysis is that morbidity was evaluated prospectively using internationally validated criteria to record outcome. ${ }^{12,13}$ This study confirms that delayed resection and reconstruction after damage control is feasible with a 
reasonable prospect of survival. We have demonstrated that a PPPD is entirely appropriate and that pylorogastric resection is only necessary when dictated by the extent of injury. A practical argument has been advanced that a pancreatogastrostomy may be a safer option than a pancreatojejunostomy when conventional anastomoses are high-risk due to edematous tissues. These techniques do not need to be used in all pancreatoduodenal injuries requiring resection, but should be applied and adapted to the severity of the situation. Our data emphasize that these are complex cases with significant postoperative morbidity and are best managed collaboratively by trauma and Hepatobiliary and Pancreatic (HPB) surgical teams.

\section{References}

1. Krige JE, Navsaria PH, Nicol AJ. Damage control laparotomy and delayed pancreatoduodenectomy for complex combined pancreatoduodenal and venous injuries. Eur J Trauma Emerg Surg 2016;42:225-30.

2. Wang GF, Li YS, Li JS. Damage control surgery for severe pancreatic trauma. Hepatobiliary Pancreat Dis Int 2007;6:56971.

3. Krige JE, Nicol AJ, Navsaria PH. Emergency pancreatoduodenectomy for complex injuries of the pancreas and duodenum. HPB (Oxford) 2014;16:1043-9.

4. Chrysos E, Athanasakis E, Xynos E. Pancreatic trauma in the adult: current knowledge in diagnosis and management. Pancreatology 2002;2:365-78.

5. Subramanian A, Dente CJ, Feliciano DV. The management of pancreatic trauma in the modern era. Surg Clin North Am 2007;87:1515-32.

6. Farrell RJ, Krige JE, Bornman PC, et al. Operative strategies in pancreatic trauma. Brit J Surg 1996;83:934-7.

7. Krige JE, Kotze UK, Hameed M, et al. Pancreatic injuries after blunt abdominal trauma: an analysis of 110 patients treated at a level 1 trauma centre. S Afr J Surg 2011;49:62-4.

8. Chinnery GE, Krige JE, Kotze UK, et al. Surgical management and outcome of civilian gunshot injuries to the pancreas. Brit J Surg 2012;99:140-8.

9. Krige JEJ, Bornman PC, Terblanche J. The role of pancreatoduodenectomy in the management of complex pancreatic trauma. In: Hanyu F, Takasaki K, eds. Pancreatoduodenectomy. Tokyo: SpringerVerlag; 1997. pp 49-62.

10. Krige JE, Kotze UK, Nicol AJ, Navsaria PH. Morbidity and mortality after distal pancreatectomy for trauma: a critical appraisal of 107 consecutive patients undergoing resection at a level 1 trauma centre. Injury 2014;45:1401-8.

11. Moore EE, Cogbill TH, Malangoni MA, et al. Organ injury scaling, II: pancreas, duodenum, small bowel, colon, and rectum. J Trauma 1990;30:1427-9.

12. Bassi C, Dervenis C, Butturini G, et al. International study group on pancreatic fistula definition. Postoperative pancreatic fistula: an international study group (ISGPF) definition. Surgery 2005;138:8-13.

13. Clavien PA, Barkun J, de Oliveira ML, et al. The Clavien-Dindo classification of surgical complications: five-year experience. Ann Surg 2009;250:187-96.

14. Terblanche J, Worthley CS, Spence RA, Krige JE. High or low hepaticojejunostomy for bile duct strictures? Surgery 1990;108:828-34.

15. Hofmeyr S, Krige JEJ, Bornman PC, Beningfield SJ. A cost analysis of operative repair of major laparoscopic bile duct injuries. SAMJ 2015;105:454-7.

16. Imanaga $\mathrm{H}$. A new method of pancreaticoduodenectomy designed to preserve liver and pancreatic function. Surgery 1960;47:577-86.

17. Koniaris LG, Mandal AK, Genuit T, Cameron JL. Two-stage trauma pancreaticoduodenectomy: delay facilitates anastomotic reconstruction. J Gastrointest Surg 2000;4:366-9.

18. Thompson CM, Shalhub S, DeBoard ZM, Maier RV. Revisiting the pancreaticoduodenectomy for trauma: a single institution's experience. J Trauma Acute Care Surg 2013;75:225-8.

19. Yeo CJ, Cameron JL, Maher MM, et al. A prospective randomized trial of pancreaticogastrostomy versus pancreaticojejunostomy after pancreaticoduodenectomy. Ann Surg 1995;222:580-92.

20. Tajima Y, Kuroki T, Tsuneoka N, et al. Anatomy-specific pancreatic stump management to reduce the risk of pancreatic fistula after pancreatic head resection. World J Surg 2009;33:2166-76.
21. Krige JE, Thomson SR. Operative strategies in pancreatic trauma - keep it safe and simple. S Afr J Surg 2011;49:106-9.

22. Madiba TE, Thomson SR. Restoration of continuity following pancreaticoduodenectomy. Brit J Surg 1995;82:158-65.

23. Lermite E, Pessaux P, Brehant 0, et al. Risk factors of pancreatic fistula and delayed gastric emptying after pancreaticoduodenectomy with pancreaticogastrostomy. J Am Coll Surg 2007;204:588-96.

24. McKay A, Mackenzie S, Sutherland FR, et al. Meta-analysis of pancreaticojejunostomy versus pancreaticogastrostomy reconstruction after pancreaticoduodenectomy. Brit J Surg 2006;93:929-36.

25. Payne RF, Pain JA. Duct-to-mucosa pancreaticogastrostomy is a safe anastomosis following pancreaticoduodenectomy. Brit J Surg 2006;93:73-7.

26. Sikora SS, Posner MC. Management of the pancreatic stump following pancreaticoduodenectomy. Brit J Surg 1995;82:1590-7.

27. Delcore R, Stauffer JS, Thomas JH, Pierce GE. The role of pancreatogastrostomy following pancreatoduodenectomy for trauma. J Trauma 1994;37:395-400.

28. Topal B, Fieuws S, Aerts R, et al. Belgian Section of Hepatobiliary and Pancreatic Surgery. Pancreaticojejunostomy versus pancreaticogastrostomy reconstruction after pancreaticoduodenectomy for pancreatic or periampullary tumours: a multicentre randomised trial. Lancet Oncol 2013;14:655-62.

29. Que W, Fang H, Yan B, et al. Pancreaticogastrostomy versus pancreaticojejunostomy after pancreaticoduodenectomy: a meta-analysis of randomized controlled trials. Am J Surg 2015;209:1074-82.

30. Duconseil P, Turrini 0, Ewald J, et al. Biliary complications after pancreaticoduodenectomy: skinny bile ducts are surgeons' enemies. World J Surg 2014;38: 2946-51.

31. Northover JM, Terblanche J. A new look at the arterial supply of the bile duct in man and its surgical implications. Brit J Surg 1979;66:379-84. 